\title{
Towards AI-based Interactive Game Intervention to Monitor Concentration Levels in Children with Attention Deficit
}

\author{
Diego R. Faria, Jordan J. Bird, Cintia Daquana, Jhonatan Kobylarz, and Pedro P. S. Ayrosa
}

\begin{abstract}
Preliminary results to a new approach for neurocognitive training on academic engagement and monitoring of attention levels in children with learning difficulties is presented. Machine Learning (ML) techniques and a Brain-Computer Interface (BCI) are used to develop an interactive AI-based game for educational therapy to monitor the progress of children's concentration levels during specific cognitive tasks. Our approach resorts to data acquisition of brainwaves of children using electroencephalography (EEG) to classify concentration levels through model calibration. The real-time brainwave patterns are inputs to our game interface to monitor concentration levels. When the concentration drops, the educational game can personalize to the user by changing the challenge of the training or providing some new visual or auditory stimuli to the user in order to reduce the attention loss. To understand concentration level patterns, we collected brainwave data from children at various primary schools in Brazil who have intellectual disabilities e.g. autism spectrum disorder and attention deficit hyperactivity disorder. Preliminary results show that we successfully benchmarked (96\%) the brainwave patterns acquired by using various classical ML techniques. The result obtained through the automatic classification of brainwaves will be fundamental to further develop our full approach. Positive feedback from questionnaires was obtained for both, the AI-based game and the engagement and motivation during the training sessions.
\end{abstract}

Index Terms-Technology for educational therapy, children with attention deficit, BCI, AI-based games, machine learning.

\section{INTRODUCTION}

Over the past years, games have become a technological service tool in different application domains. Some projects combine a direct application of games as therapeutic tools in healthcare for rehabilitation and development of cognitive function. Experiments with children demand the design of the interaction functionalities and interface for ease of use. It has been proven that games can boost confidence and interest in learners who otherwise have lack of confidence in classrooms. Gaming can be used to encourage engagement and motivation as well as promote learning if applied properly to areas in which they would be most beneficial.

Undoubtedly, technology in education helps individuals

Manuscript received March 16, 2020; revised June 6, 2020

Diego R Faria and Jordan J. Bird are with ARVIS Lab, Computer Science Department, School of Engineering and Applied Science, Aston University, UK (e-mail: \{d.faria. birdj1\}@ aston.ac.uk).

Cintia Daquana is with Education Secretariat of Cambe-PR Brazil, Department of Special Education and Inclusion, Brazil (e-mail: educacaoinclusiva@cambe.pr.gov).

Jhonatan Kobylarz is with Federal University of Parana, Brazil.

Pedro Ayrosa is with LABTED-UEL and Computer Science, Londrina State University, Brazil (e-mail: ayrosa@uel.br). develop necessary skills, but it also opens up opportunities for a much more fluid learning experience. The need to deliver continuous improvement, are driving schools and higher education institutions to seek out proven new ways of delivering learning, including people with special needs. By combining technology with traditional forms of teaching, learning providers can attain powerful means of achieving results, while benefitting from a strong return on investment. Whether cost savings are a primary or additional objective, technology opens up a realm of opportunities for new and improved content delivery and personalized learning. Technology can be personalized to meet each person's needs through a blend of cognitive-motor gaming functions. In addition, parents have the independence to have their children learn within and outside clinical or school sessions by using this technology on mobile devices. In the long term, it is a worthwhile investment (e.g. training teachers / educators of students with special needs), with tools having a proven record of maximizing children engagement. Games can be a decisive vehicle through which children learn about themselves, the environment, and develop social skills. However, for many children with any kind of impairment, adapted play opportunities are often limited.

AI-based interactive games can be applied to child-machine interaction within the context of educational therapy for assisting the facilitation of adaptive learning-related coping and improved cognitive skill outcomes in educational settings, focusing on aiding children with learning difficulties. The application of technology to this intervention is a promising and ground-breaking avenue to promote adjustment and development in children, who tend to be increasingly enthusiastic about the use of this technology.

In this work we introduce a new approach for an intelligent system by building an adaptive AI-based game for neurocognitive training to boost and maintain the concentration levels of children with intellectual disability and attention deficit. Preliminary work using ML techniques combined with BCI, and a Natural User Interface (NUI) for tracking hand motion, makes this game more attractive for children, engaging them to this kind of technology, awakening their interest and motivation to play educational games. During the playing session we recorded and measured children's brainwave patterns to monitor their concentration level, towards adapting the game interface when the concentration drops, by stimulating them through some visual or auditory stimuli on the interface to keep their attention and focus on the training session. Fig. 1 and Fig. 2 depict a sketch of our proposed approach in terms of 
technological development and future implementation steps.

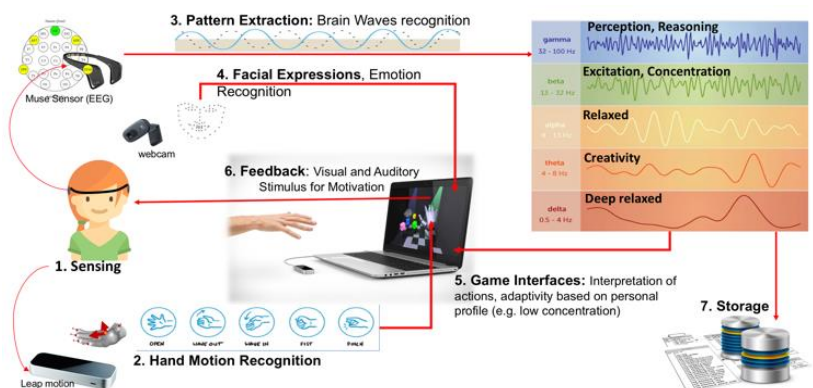

Fig. 1. Overview of the proposed AI-based Interactive Game (full system) using EEG brain waves to classify concentration levels, hand motion for gesture recognition to control the game, and emotion recognition to analyze the mood of the children. The multimodal data will help the system to adapt to the current state of the users to keep them motivated/concentrated.

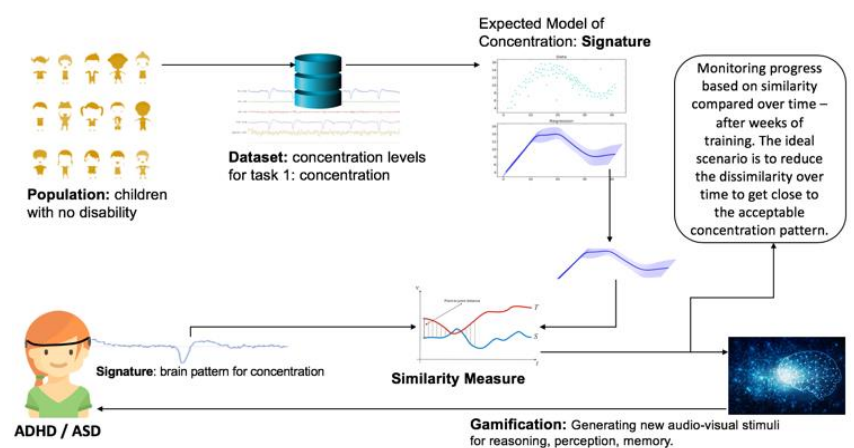

Fig. 2. Overview of the proposed approach to monitor the progress of children over time by using our neurocognitive training through AI-based games.

The remainder of this paper is as follows: Section II describes Related Work, Section III presents the technological development and methods used for the intelligent game implementation, Section IV describes the apparatus and experimental setup for data acquisition, Section V presents the preliminary results followed by Section VI with discussions and strategy for future improvements and finally, Section VII draws the conclusions.

\section{BACKGROUND AND RELATED WORK}

Games can provide a better and more efficient learning environment as they introduce a 'fun' element to education which makes the whole experience more appealing for students. Gamification of education is not only there to engage users but also to develop cognitive abilities as well as problem solving skills. The lack of enjoyment in education leads to failure in learning [1]. When students are engaged, their motivation to learn will increase because they become more focused [2]. Students thrive on dynamic learning experiences. Boredom is still a challenge in traditional education settings. The view of combining gaming with education is not a new one, however recently, there has been a steady increase in "gamifying" education and creating more interactable content to motivate learners and increase engagement [3].

A study conducted by [4] which investigated how the role of modern games in children's development concluded that games with enough dynamic content can enable children to develop physically their motor skills and intellectually through problem solving skills. There is a strong association between engaging in computer-based learning activities and cognitive development in children. Research conducted by [5] found the evidence that specially designed games do have benefits in increasing coordinated motor skills. Authors in [6] found strong evidence in measurable changes of neural processing through the use of gaming. It is important to note that enhanced cognitive performance is not a product of all the digital games that are available, but the essence of increasing cognitive processing through games is a possibility, if designed correctly. There are several ways to assess the usability and efficacy of educational games. Human-Computer Interaction (HCI) techniques, (e.g. semiotic inspection), allow computer scientists and educational game developers to evaluate such games and efficacy on children development [7].

The authors in [8] designed an interesting and novel interface integrating a commercial headband with one single dry electrode to get beta frequency from the frontal lobe to measure the concentration level. They integrated the virtual environment with the leap motion technology for gesture recognition. They aimed at using this novel interface developed in Unity for children with cerebral palsy. Despite the idea being interesting, the researchers did not use any machine learning concepts for gesture recognition with the leap motion sensor, nor to automatically classify brainwave activity from the EEG sensor. Instead, this work opted for basic functionalities provided by both commercial sensors as tools, and thus analysis of the performance of concentration levels of the users or the efficacy of the gesture recognition in specific tasks was not possible. However, they evaluate the acceptance of their novel virtual environment as a potential user-friendly interface that can be used to measure concentration level.

Different approaches have been developed using EEG data to classify emotions [9], immersive 3D games [10], control games [11], [12], and analysis of brainwave patterns of concentration, mood, etc. while patients play games [13]. But none of them have combined an intelligent game based on response of EEG, natural user interface and emotional reactions together to keep the user's concentration during the interaction. Furthermore, none of them have monitored and analyzed long-term data to monitor the progress of the user over time after multiple training sessions. This work is going beyond state-of-the-art by developing an intelligent system that will be able to adapt to the users by generating appropriate stimuli to keep their concentration during the training sessions based on the classification of EEG brainwave patterns. In addition, the future implementation shown in Fig. 2 will make our approach a potential tool for neurocognitive training of children with attention deficit.

\section{AI-BASED INTELLIGENT GAME DEVELOPMENT}

The idea of an AI-based interactive game is conceptualized here for an intervention context, with a number of potential applications in the education system, such as cognitive educational therapy (e.g. facilitation of coping skills), and health promotion settings (e.g. training of health-related skills). Current empirical evidence suggests that the use of 
smart technology in therapeutic and educational context is likely to enhance the outcomes of cognitive-behavioral interventions. The primary question of this work is: How can one endow an intelligent game with advanced perception, learning and interaction capabilities in order to stimulate children with learning difficulties to enhance their cognitive and motor functions without stressing, but engaging them? In order to answer this question, our strategy is focused on: (i) the development of an AI-based game which adopts an approach to automatically classify mental states to measure concentration levels through EEG data; (ii) control and interaction via gesture recognition using hand motion patterns within a game environment; (iii) focusing the neurocognitive training using user-friendly interfaces for tasks such as reasoning, hand-eye coordination, and memory to improve the attention deficit of children with learning difficulties; (iv) real-time, autonomous adaption by the game when concentration drops. Once the concentration level is classified during the user interaction, the intelligent system can make decision on whether new stimulus (visual or auditory) is needed to keep the user attention to avoid concentration loss.

\section{A. EEG Data Processing and Classification}

Our EEG classification technique is based on previous observations with mental state [14] and emotional state [15]. In these works, the temporal brainwave signals are processed, and mathematical features are extracted. This is performed through a sliding window technique, where windows of length $1 \mathrm{~s}$ with an overlap of $0.5 \mathrm{~s}$ capture short segments of brain activity, and the temporal data is then converted to singular data points covering the window through various algorithms. The 988 features derived from the $1 \mathrm{~s}$ windows as well as the $0.25 \mathrm{~s}$ and $0.5 \mathrm{~s}$ windows by offset include Fourier Transforms, Shannon Entropy, and Log-energy Entropy among many others ${ }^{1}$. An extensive set of features beyond those usually observed in a clinical setup [17], [18] are extracted due to the more cost-effective nature of the Muse headband sensor in comparison to expensive but higher quality clinical EEGs.

Following this, the features are organized into a matrix, and since temporal segments are now represented as numerical features, machine learning algorithms can learn useful pattern recognition rules from the data. In this study, we consider the ground-truth dataset; that is, the data collected during the calibration phase at the start of the experiment. We benchmark various classical machine learning algorithms on the ternary problem of concentrating/relaxed/neutral and compare the predictions to the collected data in order to discern how classifiable the data is prior to future exploration into the unknown data, which is the data recorded while the child is interacting with the educational game. Training is performed on the basis of 10 -fold cross validation technique in order to prevent overfitting.

\section{B. Interfaces Development Using UNITY Game Engine}

Unity is a 2D and 3D game development engine created by Unity Technologies, allowing the development of a wide range of games on multiple platforms. It has been extensively

${ }^{1}$ The full list of features is detailed in [14]. used in industry and academia since it has a number of features that make it particularly appealing including relevant standpoints, such as inexpensive, easy to use, has a fairly shallow learning curve, accommodates multiple scripting languages, and ships with intuitive prototyping tools. Our games are based on educational games reported in the state-of-the-art, focused on perception, memory, reasoning and attention. We designed a spelling game, where the interface randomly selects images of animals and their sound, separated into categories of easy, medium and complex spelling. Once children interact with the interface and progress on each phase, the difficulty is increased. The interface generates random cubes with alphabetic letters on the ground, and the user has to pick-up the letters in the correct order corresponding the animal's name and throw it into a box until the word is complete. If the player succeeds, then a new image of animal is randomly given, otherwise, the game warns the user about the mistake and prompts the user to try again. Fig. 3 shows an example of this interface.
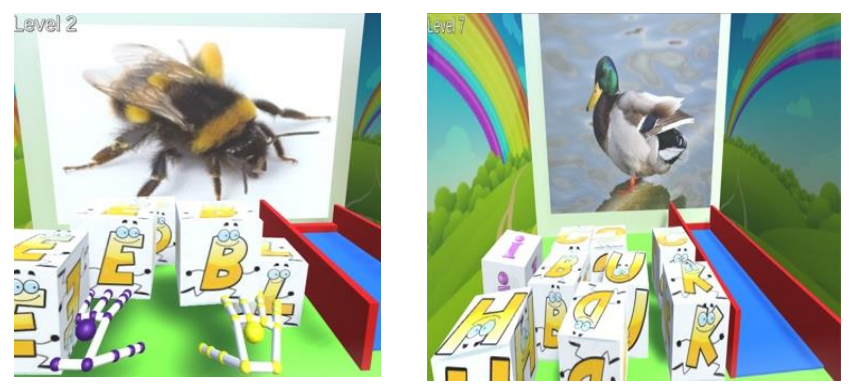

Fig. 3. Spelling game interfaces developed in Unity. The user has to pick-up the corresponding letters of the animal name and throw them to the right side (inside the box).

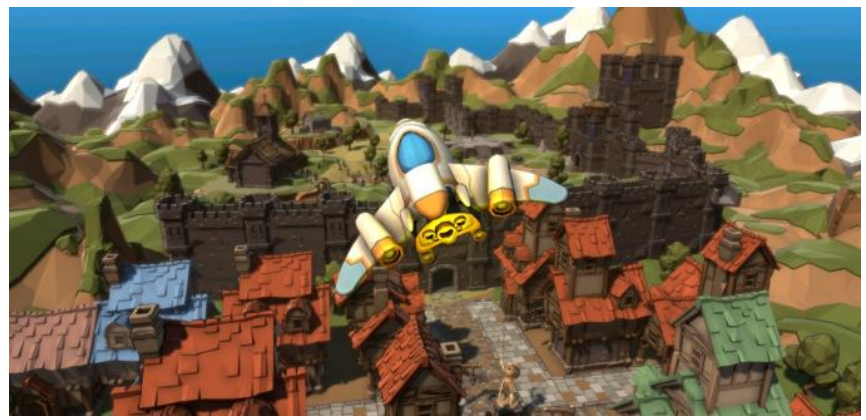

Fig. 4. Aircraft interface designed in Unity and used with leap motion to grab the user hand motion to control the aircraft.
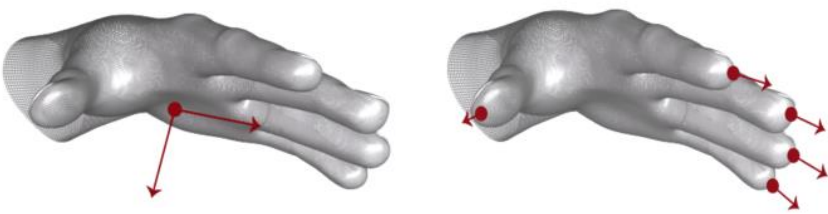

Fig. 5. Leap Motion Data. Left: Palm tracking data. Right: Fingertips tracking data

Another interface designed is an aircraft controlled by the hand, where its pose and orientation is given by the hand pose (roll-pitch-yaw), and the acceleration as well by moving the hand towards the screen or backwards, and also allowing other directions such as left and right. Fig. 4 shows the interface. The main idea of this game is to control the aircraft and avoid obstacles, find free routes, and gather objectives (coins) to increase the player's score. It demands a hand-eye coordination to follow specific routes and moving the hand 
appropriately for the correct motion within the virtual environment, with the ability to increase the difficulty (minimum speed, obstacles) if required, based on concentration levels measured from the brain.

\section{Gesture Recognition}

Our gesture recognition module is based on the Leap Motion Controller (LMC) Software Development Kit (SDK) functions. The LMC is primarily designed for hand gesture and finger position detection in interactive software applications. It consists of three infrared emitters and two CCD cameras to track the hand. It provides preprocessed data through their Application Programming Interface (API). The data provided is given as follows (Fig. 5): the position of the palm frame-by frame, its normal and velocity; the hand direction; fingertips position and velocity; and arm direction. Currently we are using the leap-motion API functions for gesture recognition instead of implementing our own classifier, since it works very well for gestures such as opening and closing the hand for simple tasks (e.g. pinch, pick-up and release an object, and also static gesture like pointing, two-fingers, three-fingers and so on). The hand pose provided by the API can be directly mapped as the controller (e.g. palm and finger position and orientation) in the game interface to move an object forward, backward, left, right and rotate it based on the hand orientation.

The Unity Game Engine and LMC SDK are easily compatible with one another, making game design and implementation integration easier, since the LMC functions are embedded within the Unity development interface.

\section{EXPERIMENTAL SETUP AND DATA ACQUISITION}

In order to gather data for the experiment, the commercial Muse headband (EEG) sensor is used as can be observed in Fig. 6. The subject group was made up of a total of 30 children from 3 different public primary schools in Cambé, a municipality within the State of Paraná in Southern Brazil. All of the children's legal parents or guardians were informed in detail of the project characteristics and written consent was given. Special monitoring roles were created for the trial in order to ensure compliance of ethical requirements, confidentiality and protection of personal data. No trials were performed without previous approval by the ethical committee and data protection authorities in Brazil. The pilot trials were conducted in accordance with the highest ethical standards from the UK and Brazil. To ensure that the information is easy to understand, all written information that was given to the involved people was approved by experts on "Easy to Read" guidelines. All trials were conducted after receiving the signed "Informed Consent Form". The sample size of 30 children is selected for preliminary experimentation, with positive results presenting assurance and reason for collecting a much larger dataset in the future.

The subject group of 30 children were aged from six to ten years old, 24 were male and 6 were female. The children considered in this experiment all had forms of disability including Intellectual Disability (ID), Autism Spectrum Disorder (ASD) and Attention Deficit Hyperactivity Disorder (ADHD) which were widespread within the subject group, oppositional defiant disorder (ODD) and Hydrocephaly were also present within the subject group. One of the subjects suffered with Cerebral Palsy. Finally, one subject with Down's Syndrome could not concentrate for a long enough period for data to be recorded, but through informal observation was seen to enjoy the Leap Motion game. Due to this, another child was added to the subject group to retain the planned 30 (the above ethical consent was obtained). An example of brain activity being recorded can be seen in Fig. 7, and the full experimental setup can be seen in Fig. 8. For each of the children, up to two minutes of data is recorded during the interaction with games for each of the three states of interest by the experimental setup shown in Fig. 8. For states of concentration, several of the subjects were not recorded for the full two minutes due to issues arising with attention for the duration of the recording. This leads to a slight class imbalance of 820 data objects for concentrating and 830 for both relaxed and neutral data. Although this slight imbalance is present, it was noted that Precision and Recall strongly supported the observed accuracy of the algorithms, detailed further in Section V. The calibration and educational activities can be seen in Fig. 9. For the concentration state, children played the 'shell game' with an instructor. The child was tasked with following the location of a ball when passed under three cups, with difficulty increasing based on the child's performance in order to retain attention through challenge. For the relaxed state, the child was asked to breathe slowly (following the instructor) and to sit in a position which allowed for relaxation of muscles. For the neutral state, no stimuli were present. The data was recorded based on observation, if the subject were to lose concentration on the task, then the recording would be stopped. This was done in order to prevent contamination of the calibration data.

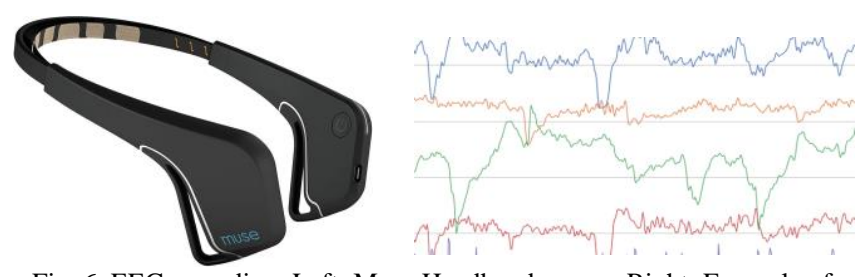

Fig. 6. EEG recording. Left: Muse Headband sensor. Right: Example of temporal data (alfa, beta, theta and gamma signals provided by the sensor).

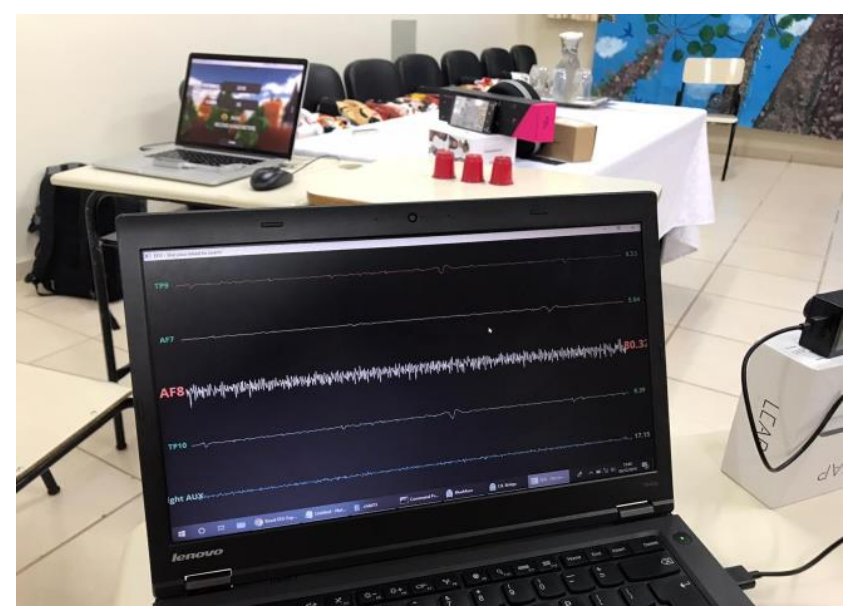

Fig. 7. Brain activity observed by an instructor. The child has been omitted from the photo for privacy reasons. Note that this data is not being recorded, since the child is behind the camera attempting to make the headband comfortable. Prior to data being recorded, the instructor made sure the headband was properly calibrated and recording normally. 


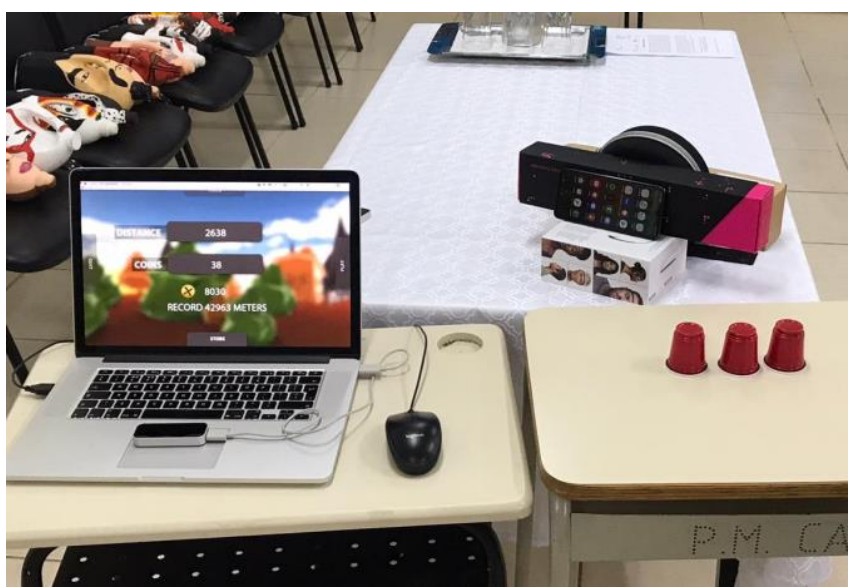

Fig. 8. Example of the experimental set up. On the left table, the Leap Motion sensor on top of the laptop and game are ready. On the right, the Shell Game is set up for calibration data as well as a smartphone used to record videos for future extraction of facial features to classify emotions etc
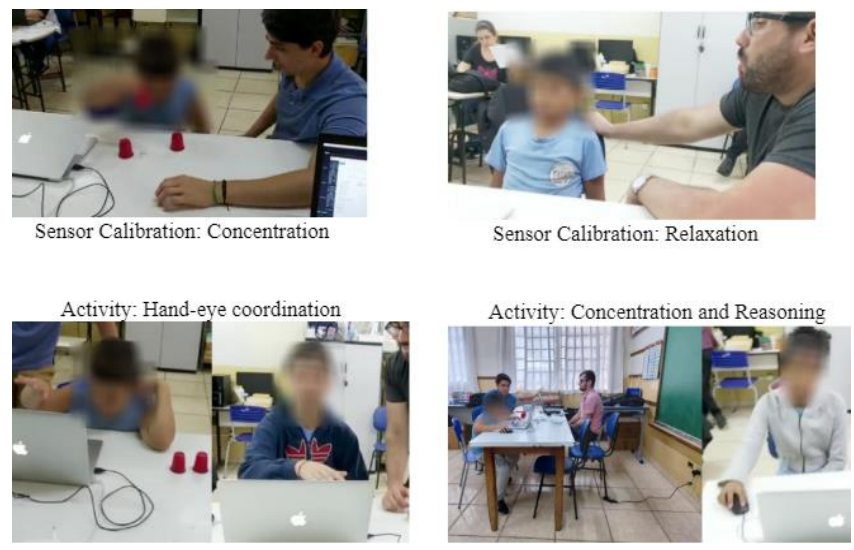

Fig. 9. Data Acquisition: Examples of the calibration and educational activities using the games for interaction. Faces are blurred for privacy reasons.

Following the calibration experiment, all the children played two educational games - one with the Leap Motion and one without, using the mouse to select objects starting with a specific letter of the alphabet given by the game interface. In future we plan to take the calibrated model derived in this experiment and apply it to this data in order to discern the effects of physical engagement in games.

\section{PREliminary RESUlts}

At this stage, the results are related to the performance of classification algorithms given the EEG data. The results from the single algorithm benchmarking experiment can be observed in Table I. Random Forest and Logistic Regression hyperparameters are manually optimized in Fig. 10 and Fig. 11. For the three performance measures, Logistic Regression seemed to be the best with an overall classification accuracy of $96.24 \%$ given three classes: concentrated, relaxed and neutral. The Logistic Regression model took 130 seconds to execute whereas the Random Forest model took only 19 seconds, and thus the reduction of $0.34 \%$ overall score may be worth the reduction in computational complexity in future when the algorithm is required to run on standard office machines. In Table II, it can be observed that when combining the two best models, a higher classification accuracy is achieved for the dataset. This is observed through both two voting methods and stacking, where the voting method achieves the highest classification accuracy for the dataset. It must be noted that the models are complex and take a lengthy amount of time, around 10 to 30 minutes, on a high-end computer (Intel core i7@3.7GHz). When the two models vote on class both by average and maximum probability, scores of $97 \%$ are achieved.

TABLE I: BENCHMARKING OF MACHINE LEARNING ALGORITHMS FOR ClASSIFICATION OF CALIBRATION DATA (BRAINWAVES)

\begin{tabular}{llll}
\hline \hline Algorithm & Accuracy (\%) & Precision & Recall \\
\hline $\begin{array}{l}\text { Random Forest } \\
\text { SVM classifier }\end{array}$ & 95.9 & 0.96 & 0.959 \\
$\begin{array}{l}\text { Logistic } \\
\text { Regression }\end{array}$ & $\mathbf{9 6 . 2 4}$ & 0.934 & 0.934 \\
$\begin{array}{l}\text { Bayesian } \\
\text { Network }\end{array}$ & 82.25 & $\mathbf{0 . 9 6 2}$ & $\mathbf{0 . 9 6 2}$ \\
$\begin{array}{l}\text { 10-Nearest } \\
\text { Neighbour }\end{array}$ & 88.75 & 0.823 & 0.823 \\
\hline \hline
\end{tabular}

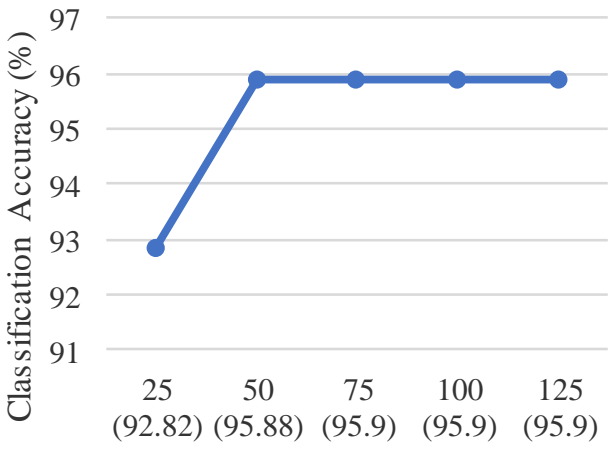

Trees in the Random Forest

Fig. 10. Random Forest Tuning: The number of Random Trees in the Forest are stepped from 25 to 125 in order to select the best hyperparameters.

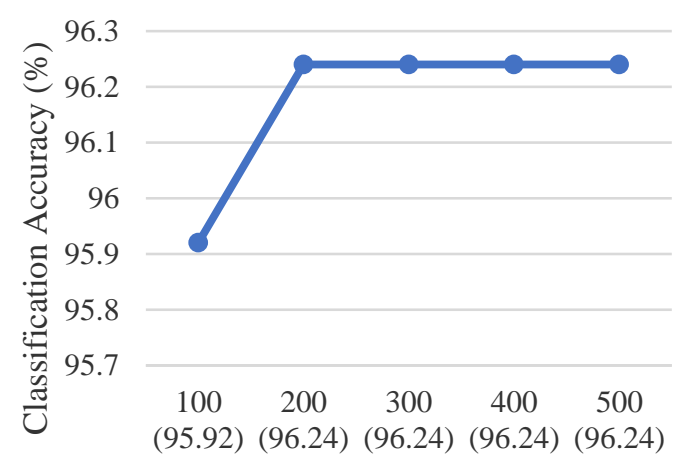

Boosting Iterations

Fig. 11. Logistic Regression Tuning: The number of boosting iterations is stepped from 100 to 500 in order to select the best hyperparameters.

TABLE II: METHODS OF ENSEMBLE FOR THE TwO BEST MODELS, LOGISTIC REGRESSION AND RANDOM FOREST TO CLASSIFY BRAINWAVES

\begin{tabular}{llll}
\hline \hline Algorithm & Accuracy (\%) & Precision & Recall \\
\hline $\begin{array}{l}\text { Vote } \\
\text { (Avg. Prob) }\end{array}$ & $\mathbf{9 7 . 0 1}$ & $\mathbf{0 . 9 7}$ & $\mathbf{0 . 9 7}$ \\
$\begin{array}{l}\text { Vote } \\
\text { (Max. Prob) }\end{array}$ & $\mathbf{9 7 . 0 1}$ & $\mathbf{0 . 9 7}$ & $\mathbf{0 . 9 7}$ \\
Stacking & 96.84 & 0.96 & 0.96 \\
\hline \hline
\end{tabular}

If the neutral state is not considered, only 2 data objects from relaxed and 1 from concentrating were misclassified as one another by the Logistic Regression model for the whole 
dataset, showing that the classification ability of the two classes is strong. In terms of the best ensemble methods, only 1 data object was misclassified. A preliminary attribute selection search given the brainwave features was performed and sorted based on attribute Information Gain (relative entropy). All attributes were noted to have at least some form of prediction value. The highest were eigenvalues and covariance matrices of all temporal windows which had an Information Gain value of around 0.6, this is visualized on a per-class basis in Fig. 12. The lowest attribute was the skewness (statistical moments) of the first quarter window which had a small value of 0.006 . Computational complexity could likely be reduced in future by performing dimensionality reduction of the benchmark matrix as well as the minimization of data extracted from brainwave activity to be classified in real time.

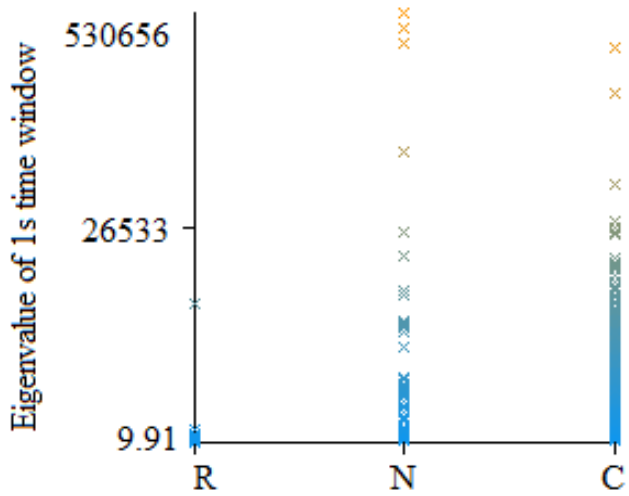

Fig. 12. Visualization of the highest relative entropy score of an attribute (Eigenvalue $\mathrm{IG}=0.685$ ) mapped to the three-class problem.

\section{DisCUSSION AND FUTURE WORK}

In this work, we successfully benchmarked various classical machine learning techniques on the dataset acquired in primary schools. In future, we plan on benchmarking a deep neural network through the same technique as [16]. Additionally, the complexity of the model was high due to the consideration of 988 numerical features, in future we will attempt to reduce the complexity of the dataset by performing and benchmarking dimensionality reduction algorithms. This will aim to improve the speed of classification without the loss of accuracy and will aid in real-time classification.

Through the derivation of a less complex model which can classify in real time, education can then be customized for the individual based on brain activity. The educational game will consider the predictions by the model as additional input to the child's physical activity, allowing for the activities to react in real time to a drop-in concentration, in order to raise the concentration level and improve the educational experience.

We will also use our educational framework as a long-term form of cognitive behavioral therapy for certain conditions. To do this, we plan on repeating the experiment in this study for a group of children who do not suffer with intellectual disability and generating a signature to describe the wave activity (see Fig. 2). Over a period of months of using our proposed framework, the children with intellectual disabilities will periodically have a similarity measure generated between their own signature and the signature generated from the non-intellectually disabled. The goal therein is to aim to increase the similarity metric over time through engaging educational activities, with real-time classification guiding the quality of individual sessions.

The planned activities outlined in this section are now enabled due to the strong classification ability of the models derived from this study which pave the way for more in-depth studies and the development of the educational framework.

In regard to evaluation of usability and acceptance of children with our developed games, where they have to wear the headband for BCI and also interact with a NUI via LMC, we applied a questionnaire to record the opinions of users after the experiments were all complete as shown below:

a) How easy was it to play the game?

b) What game interface is easier to interact with: (1) leap motion controller or (2) the traditional game using the computer mouse/pad?

c) Which game did you prefer?

d) Has been difficult or uncomfortable to wear the Muse EEG headband (BCI)?

e) Was it comfortable to wear the EEG for a long period?

f) Would you agree that you enjoyed playing the leap motion games?

g) What did you like about these games?

h) What did you dislike about these games?

i) Would you play these games again?

For a) $83.3 \%$ of the responses stated it was as easy; $10 \%$ as medium and $6.7 \%$ difficult; b) $86.7 \%$ of the children pointed that the leap motion controller is easier to use than mouse/pad; c) $83.3 \%$ of children chose the aircraft game as their favorite and the other $16.7 \%$ is split into the other games; d) $93.3 \%$ of the children answered no, it is not difficult to wear the EEG sensor; e) $96.7 \%$ of the children answered yes; f) $100 \%$ agreed; g) $83.3 \%$ answered the NUI (using the hand to control the game); h) in this question, it is interesting to point out that $53.3 \%$ mentioned that after long time playing with leap motion, sometimes they got tired to keep the hand up to control the aircraft; i) $100 \%$ yes.

It is important to mention here that all children, with their disabilities, some of them even with motor disability, got well-engaged with the game interfaces. Most of them wanted to continue playing after their sessions. The educational staff observed and pointed out that even the most difficult students in terms of attention deficit (specifically ODD) were focused on the games interface. They noticed that with only a few instructions the children were able to play the games, and through intuition they realized how to solve specific problems, by observing us (instructors demonstrating only once how to play in a short of period of time). Since most of the children in these schools we visited were children belonging to low-income families, some of them had never played a similar type of game before. Most of them showed high anxiety to participate on these experiments, since they were told a few days before the experiments, and they were very motivated and anxious waiting for the day.

The next step is to check the confidence given by the concentration level classification to trigger the game to adapt to the user and change the stimuli in case the concentration level drops below a certain threshold. This functionality is implemented, waiting for the real-time classification of the EEG data, that is currently being developed. Following this step, we will implement the approach mentioned in Fig. 2, as a final version of our artificially intelligent game to monitor 
the progress of children over time at schools. With all validation done so far, we can affirm that this approach has potential to have strong societal impact in children with attention deficit towards improving their cognitive abilities.

\section{CONCLUSION}

This work introduces a new approach for neurocognitive training towards improving children's cognition and concentration by using intelligent games. We combined BCI to measure concentration levels and engagement of children during the interaction with the game and a NUI controlled by hand gestures. Data acquisition of brainwave patterns of different groups of children with disability or disorder (e.g. ID, ODD, ASD, ADHD) were acquired, so that we can check their patterns of concentration level when performing multiple tasks. Preliminary results on children brainwaves classification of concentration show that our framework achieved $96 \%$ accuracy. Questionnaires pointed out positive feedback on the use of the game and acceptance of children to this technology in this kind of intervention. Future work will address our system fully integrated, enabling the game ability to learn the user concentration pattern and make decisions to generate new stimuli when the user's attention drops, effectively enabling the real-time individual personalization of education.

\section{CONFLICT OF INTEREST}

The authors declare no conflict of interest.

\section{AUTHOR CONTRIBUTIONS}

D. Faria came up with the idea and proposal for this new approach and defined the mathematical models of features extraction for EEG brainwaves. D. Faria has also collaborated on the games interface design. J. Bird defined and tested the classification and optimization models. J. Bird contributed with the system implementation and integration, e.g. sensors, games and machine learning. C. Daquana and J. Kobylarz helped to conduct the pilots with children, interacting with them and explaining the technology prior to the experiments. They prepared and applied the questionnaires as well. P. Ayrosa helped with discussions and feedback on each phase of the project, including the theoretical methods and research practice. D. Faria, J. Bird, C. Daquana and P. Ayrosa looked at the ethical process. D. Faria and J. Bird were in charge of writing, but all authors provided feedback and contributed with specific sessions in this paper.

\section{ACKNOWLEDGMENT}

This work is partially supported by Fundação Araucária Paraná / CONFAP Brazil and the Royal Society UK / Newton Fund, through a mobility grant awarded to Dr Diego Faria and Prof. Pedro Ayrosa for the project: "Engagement through AI-based Interactive Games: Neurocognitive training for children with learning difficulties" in 2019. We would like to especially thank the Municipal Education Secretariat of Cambé-PR, Brazil, in particular, Claudia S. C. Segura, for the kind assistance and help in conducting the experiments with children in local primary schools in Cambé-PR, and the directors of these schools: Irma Hilda Soares Municipal
School; Padre Symphoriano Kopf Municipal School; and Professor Jacídio Correia Municipal School.

\section{REFERENCES}

[1] D. J. Shernoff, M. Csikszentmihalyi, B. Schneider, and E. S. Shernoff, "Student engagement in high school classrooms from the perspective of flow theory," School Psychology Quarterly, vol. 18, no. 2, pp 158-176, 2003.

[2] I. Glover, "Play as you learn: Gamification as a technique for motivating learners," in Proc. W. Conf. on Edu., Hyperm. and Telecom., 2013.

[3] C. Pappas. (2013). Gamify the classroom. [Online]. Available: http://elearningindustry.com/gamify-the-classroom

[4] S. Petrovska, D. Sivevskaa, and O. Cackov, "Role of the game in the development of preschool child," Social and Behav. Sci, p. 92, 2013.

[5] N. Pierce. State-of-the-art report: Digital game-based learning for early childhood. [Online]. Available: https://www.learnovatecentre.org/

[6] I. Granic, A. Lobel, and R. Engels, "The benefits of playing video games," American Psychologists, 2014.

[7] A. Brandao, D. G. Trevisan, L. Brandao, B. Moreira, G. Nascimento, C. N. Vasconcelos, E. Clua, and P. T. Mourao, "Semiotic inspection of a game for children with down syndrome," in Proc. Brazilian Symposium on Computer Games and Digital Entertain, 2010.

[8] J. M. Oliveira, R. Carneiro, G. Fernandes, C. S. Pinto, P. R. Pinheiro, S. Ribeiro, and V. H. C. Albuquerque, "Novel virtual environment for alternative treatment of children with cerebral palsy," Comput Intell Neurosci., 2016.

[9] G. Chanel, C. Rebetez, M. Bétrancourt, and T. Pun, "Emotion assessment from physiological signals for adaptation of game difficulty," IEEE Trans. Syst. Man Cybern. A Syst. Hum., vol. 41, pp. 1052-1063, 2011

[10] E. Lalor, S. Kelly, C. Finucane, R. Burke, R. Smith, R. Reilly, and G. McDarby, "Steady-state VEP-based brain-computer interface control in an immersive 3D gaming environment," Eurasip J. Appl. Signal Process, 2005.

[11] D. Huang, K. Qian, D. Y. Fei, W. Jia, X. Chen, and O. Bai, "Electroencephalography (EEG)-based brain-computer interface (BCI): A 2-D virtual wheelchair control based on event-related desynchronization/synchronization and state control," IEEE Trans. Neural Syst. Rehabil. Eng., vol. 20, pp. 379-388, 2012.

[12] A. Finke, A. Lenhardt, and H. Ritter, "The MindGame: A P300-based brain-computer interface game," Neural Netw., vol. 22, pp. 1329-1333, 2009.

[13] C. Russoniello, K. O’Brien, and J. Parks, "The effectiveness of casual video games in improving mood and decreasing stress," J. Cyber Ther. Rehabil., vol. 2, pp. 53-66, 2009.

[14] J. J. Bird, L. J. Manso, E. P. Ribeiro, A. Ekart, and D. R. Faria, "A study on mental state classification using EEG-based brain-machine interface," in Proc. International Conference on Intelligent Systems, 2018.

[15] J. J. Bird, A. Ekart, C. D. Buckingham, and D. R. Faria, "Mental emotional sentiment classification with an eeg-based brain-machine interface," in Proc. Int. Conf. on Digital Image and Signal Processing (DISP'19), 2019.

[16] J. J. Bird, D. R. Faria, L. J. Manso, A. Ekárt, and C. D. Buckingham, "A deep evolutionary approach to bioinspired classifier optimisation for brain-machine interaction," Complexity, 2019

[17] R. P. Brenner and N. Schaul, "Periodic EEG patterns: Classification, clinical correlation, and pathophysiology," Journal of Clinical Neurophysiology: Official Publication of the American Electroencephalographic Society, vol. 7, no. 2, pp. 249-267, 1990.

[18] N. F. Güler, D. U. Elif, and I. Güler. "Recurrent neural networks employing Lyapunov exponents for EEG signals classification," Expert Systems with Applications, vol. 29, no. 3, pp. 506-514, 2005.

Copyright (C) 2020 by the authors. This is an open access article distributed under the Creative Commons Attribution License which permits unrestricted use, distribution, and reproduction in any medium, provided the original work is properly cited (CC BY 4.0).

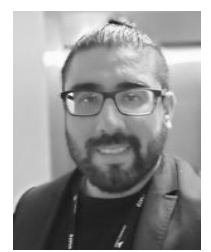

Diego R. Faria is a senior lecturer in computer science. $\mathrm{He}$ is with the School of Engineering and Applied Science, Aston University, Birmingham (UK). He is the coordinators and founder of the ARVIS Lab (Aston Robotics, Vision and Intelligent Systems). Currently (2019-2022) he is the project coordinator of EU CHIST-ERA InDex project (Robot In-hand Dexterous manipulation by extracting data from human manipulation of objects to improve robotic autonomy and dexterity) funded 
by EPSRC UK. Dr Faria is also PI and Co-I (2020-2022) of two projects with industry (KTP-Innovate UK scheme) related to perception and autonomous systems applied to autonomous vehicles, and NLP and image processing for multimedia retrieval. He received his Ph.D. degree in electrical and computer engineering from the University of Coimbra, Portugal, in 2014. He holds an M.Sc. degree in computer science from the Federal University of Parana, Brazil, in 2005. In 2001, he earned a bachelor's degree in informatics technology (data computing \& information) and he has finished a computer science specialization in 2002 at Londrina State University, Brazil. From 2014 to 2016 he was a postdoctoral fellow at the Institute of Systems and Robotics, University of Coimbra where he collaborated on different projects funded by EU commission and the Portuguese government in areas of Robot Grasping, Perception, Cognitive Robotics and Assisted Living. His research interests are assisted living, intelligent systems, and cognitive robotics.

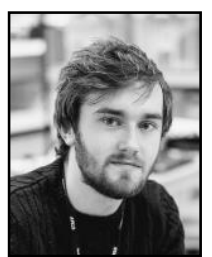

Jordan J. Bird achieved a first class bachelor's degree with honours in computer science at Aston University in the United Kingdom, before continuing with $\mathrm{PhD}$ studies at the same institution in 2018 with an awarded scholarship. Garnered through a deep scientific passion from an early age, his research interests exist largely within the field of human-robot interaction; these include, the emergence of artificial intelligence, intelligent social frameworks, turing's imitation game, deep machine learning, and transfer learning. jordan is a founding member of the aston robotics, Vision and Intelligent Systems (ARVIS) laboratory at Aston University.

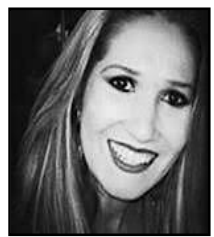

Cintia Daquana is the pedagogical coordinator at the Municipal Education Secretariat of Cambé-PR, Brazil. She is responsible for special education and inclusion of children with disabilities. She has a degree in pedagogy/education by the State University of Londrina, Brazil. She also has a certified specialization course (Lato-sensu) in pedagogical work in early childhood education (State University of Londrina, Brazil); Special Education (UNOPAR, Brazil), and Psychopedagogy (INESUL, Brazil). Her research interests are: special education, children with learning difficulties, and technology for education.

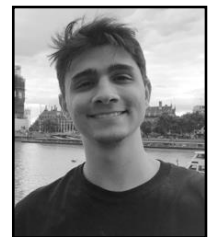

Jhonatan Kobylarz is an electronic engineering student at Universidade Federal do Paraná (UFPR), Brazil. His research interests include deep machine learning towards social robotic interaction, bioengineering and computer vision.

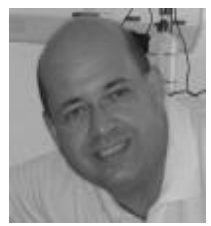

Pedro P. S. Ayrosa obtained his M.Sc. and PhD degrees in computer engineering and systems from the Federal University of Rio de Janeiro (COPPE / UFRJ) in 1992 and 2001, respectively. He earned a B.Sc. degree in mathematics from Universidade Federal Fluminense - UFF, Brazil, in 1988. He is currently an associate professor at the State University of Londrina (UEL) having worked in undergraduate, specialization and master's degrees in computer science. He was a member of the editorial board of the State University of Londrina Publisher (Eduel). He is the institutional examiner of courses and Distance Learning Education at INEP-Brazil. He is an expert in Ad-hoc distance learning at the Department of Science and Technology of the State of Parana. He is the general coordinator of the Center of Distance Education at State University of Londrina (NEAD-UEL), the local coordinator of the Open University of Brazil (UAB), and director of the Laboratory of Educational Technology (LABTED). His research interests are: distance learning technology, artificial intelligence, and neural networks. 